\title{
MS33-02 | Modulated Self-Assembly of Hard and Soft Porous Crystals
}

Forgan, Ross (University of Glasgow, Glasgow, GBR)

Metal-organic frameworks (MOFs) are network materials comprised of organic ligands connected by metal ion clusters into multidimensional structures that often have permanent porosity. Their chemically addressable structures, combined with their ability to store large quantities of small molecules within their pores, have led to applications in gas storage, heterogeneous catalysis, sensing, and drug delivery, amongst others. Coordination modulation, the addition of monomeric modulators to synthetic mixtures, can tune particle size from nanometres to centimetres, through capping of crystallites (decreasing size) or coordinative competition with ligands (increasing size).

The talk will cover the development of our own modulation techniques for a range of MOFs, describing the versatility of modulation in controlling phase purity and physical properties such as interpenetration, defectivity, and porosity. Our techniques provide access to high quality single crystals of many different MOFs, allowing the subsequent characterisation of their mechanical properties, flexibility upon guest uptake, molecular spongelike behaviour, single-crystal to single-crystal postsynthetic modification, and the development of fluorescent sensors. 